\title{
Separation of variables in quasi-potential systems of bi-cofactor form
}

\author{
Krzysztof Marciniak* \\ Department of Science and Technology \\ Campus Norrköping, Linköping University \\ 60174 Norrköping, Sweden \\ krzma@itn.liu.se \\ Maciej Błaszak ${ }^{\dagger}$ \\ Department of Physics \\ A. Mickiewicz University \\ Umultowska 85, 61-614 Poznań \\ blaszakm@main.amu.edu.pl
}

November 13, 2018

\begin{abstract}
We perform variable separation in the quasi-potential systems of equations of the form $\ddot{q}=-A^{-1} \nabla k=-\tilde{A}^{-1} \nabla \tilde{k}$, where $A$ and $\tilde{A}$ are Killing tensors, by embedding these systems into a bi-Hamiltonian chain and by calculating the corresponding Darboux-Nijenhuis coordinates on the symplectic leaves of one of the Hamiltonian structures of the system. We also present examples of the corresponding separation coordinates in two and three dimensions.
\end{abstract}

AMS 2000 Classification System: 70H06,70H20,53D17

${ }^{*}$ On leave of absence from Department of Physics, A. Mickiewicz University, Poznań, Poland.

${ }^{\dagger}$ Partially supported by KBN grant No 5P03B 00420 


\section{Introduction}

In recent years, a new constructive separability theory, based on a bi-Hamiltonian property of integrable systems, was presented. In the frame of canonical coordinates the theory was developed in a series of papers [1]-[6] (see also the review article [7]), while a general case was considered in [8] - [10].

In this paper we apply the bi-Hamiltonian separability theory to solve Hamilton-Jacobi (HJ) equations for a class of quasi-potential systems, called bi-cofactor systems, systematically studied in recent papers [11]- [15].

The structure of the paper is as follows. In Section 2 we briefly describe the one-Casimir bi-Hamiltonian separability theory in case of bi-Hamiltonian systems with quadratic in momenta constants of motion and relate this theory with the classical Stäckel theory. In Section 3 we present basic facts about quasi-potential systems and their special subclass called bi-cofactor systems. Then, in Section 4, an explicit form of transformation to separated coordinates is derived, proving the separability of all nondegenerated bi-cofactor systems. We sketch the proofs of more important results of this section independently of the general statements of Section 2 in order to make this article more self-contained, but we also point out the places where the general theory and our calculations meet. Finally, in Section 5, we illustrate the obtained formulas by some examples.

\section{Stäckel separability of one-Casimir bi-Hamiltonian chains}

Let us consider a Poisson manifold $\mathcal{M}$ of $\operatorname{dim} \mathcal{M}=2 n+1$ equipped with a linear Poisson pencil $\Pi_{\xi}=\Pi_{1}-\xi \Pi_{0}$ of maximal rank, i.e. a pair of Poisson operators (tensors) $\Pi_{i}: T^{*} \mathcal{M} \rightarrow T \mathcal{M}$ each of rank $2 n$ such that their linear combination $\Pi_{1}-\xi \Pi_{0}$ is itself a Poisson operator for any $\xi \in \mathbf{R}$ (the operators $\Pi_{0}$ and $\Pi_{1}$ are then said to be compatible).

Definition 1 A scalar function $h: \mathcal{M} \rightarrow \mathbf{R}$ is Called a Casimir function (or a Casimir) of a Poisson operator $\Pi$ acting in $\mathcal{M}$ if $\Pi \circ d h=0$.

Here and in what follows $d: C^{\infty}(\mathcal{M}) \rightarrow T^{*} \mathcal{M}$ is the operator of external derivative (gradient operator) in $\mathcal{M}$ and the symbol $\circ$ denotes the composition of mappings. If - as it often happens - the Casimir $h_{\xi}$ of the pencil $\Pi_{\xi}$ 
is a polynomial in $\xi$ of order $n$

$$
h_{\xi}=h_{n} \xi^{n}+h_{n-1} \xi^{n-1}+\ldots+h_{0}
$$

the by expanding the equation $\Pi_{\xi} \circ d h_{\xi}=0$ in powers of $\xi$ and comparing the coefficients at equal powers we obtain the following bi-Hamiltonian chain:

$$
\begin{array}{ccc}
\Pi_{1} \circ d h_{0} & = & 0 \\
\Pi_{1} \circ d h_{1} & = & \Pi_{0} \circ d h_{0} \\
& \vdots & \\
\Pi_{1} \circ d h_{n} & = & \Pi_{0} \circ d h_{n-1} \\
0 & = & \Pi_{0} \circ d h_{n}
\end{array}
$$

From (2) it follows that the functions $h_{i}$ are in involution with respect to both Poisson structures. If additionally all $h_{i}$ are functionally independent, then the chain is a Liouville integrable system.

Let us now consider a set of coordinates $\left\{\lambda_{i}, \mu_{i}\right\}_{i=1}^{n}$ and a Casimir coordinate $c^{\prime}=h_{n}$ on $\mathcal{M}$ so that $\left\{\lambda_{i}, \mu_{i}\right\}_{i=1}^{n}$ are canonical with respect to $\Pi_{0}$, so that

$$
\Pi_{0}=\left[\begin{array}{cc|c}
0 & I & 0 \\
-I & 0 & 0 \\
\hline * & 0
\end{array}\right]
$$

We can try to linearize the system (2) through a canonical transformation $(\lambda, \mu) \rightarrow(b, a)$ in the form $b_{i}=\frac{\partial W}{\partial a_{i}}, \mu_{i}=\frac{\partial W}{\partial \lambda_{i}}$, where $W(\lambda, a)$ is a generating function that solves the related Hamilton-Jacobi (HJ) equations

$$
h_{r}\left(\lambda, \frac{\partial W}{\partial \lambda}\right)=a_{r}, \quad r=0, \ldots, n .
$$

In general, the HJ equations (3) are nonlinear partial differential equations that are very difficult to solve. However, there are rare cases when one can find a solution of (3) in the separable form

$$
W(\lambda, a)=\sum_{i=1}^{n} W_{i}\left(\lambda_{i}, a\right)
$$

that turns the HJ equations into a set of decoupled ordinary differential equations that can be solved by quadratures. Such $(\lambda, \mu)$ coordinates are called 
separated coordinates. In the $(a, b)$ coordinates the flow $d / d t_{j}$ associated with every Hamiltonian $h_{j}$ is trivial

$$
\frac{d a_{i}}{d t_{j}}=0, \quad \frac{d b_{i}}{d t_{j}}=\delta_{i j}, \frac{d c^{\prime}}{d t_{j}}=0, \quad i, j=1, \ldots, n
$$

and the implicit form of the trajectories $\lambda\left(t_{j}\right)$ is given by

$$
b_{i}(\lambda, a)=\frac{\partial W}{\partial a_{i}}=\delta_{i j} t_{j}+\text { const }, \quad i, j=1, \ldots, n .
$$

Theorem 2 A sufficient condition for $(\lambda, \mu)$ to be separated coordinates for the bi-Hamiltonian chain (匀) is

$$
H_{i}\left(\lambda, \mu, c^{\prime}\right)=f_{i}\left(\lambda_{i}, \mu_{i}\right), \quad i=1, \ldots, n
$$

where

$$
H_{i}\left(\lambda, \mu, c^{\prime}\right) \equiv c^{\prime} \lambda_{i}^{n}+h_{n-1}\left(\lambda, \mu, c^{\prime}\right) \lambda_{i}^{n-1}+\ldots+h_{0}\left(\lambda, \mu, c^{\prime}\right)
$$

and $f_{i}\left(\lambda_{i}, \mu_{i}\right)$ is some smooth function of a pair of canonically conjugate coordinates $\lambda_{i}, \mu_{i}$.

We will briefly sketch the proof here. The condition (7) can be presented in a matrix form

$$
\left(\begin{array}{cccc}
\lambda_{1}^{n} & \lambda_{1}^{n-1} & \ldots & 1 \\
\lambda_{2}^{n} & \lambda_{2}^{n-1} & \ldots & 1 \\
\vdots & \vdots & \ldots & \vdots \\
\lambda_{n}^{n} & \lambda_{n}^{n-1} & \cdots & 1
\end{array}\right)\left(\begin{array}{c}
c \\
h_{n-1} \\
\vdots \\
h_{0}
\end{array}\right)=\left(\begin{array}{c}
f_{1}\left(\lambda_{1}, \mu_{1}\right) \\
f_{2}\left(\lambda_{2}, \mu_{2}\right) \\
\vdots \\
f_{n}\left(\lambda_{n}, \mu_{n}\right)
\end{array}\right) \text { or } w h=f
$$

which is called a generalized Stäckel representation. Multiplying the HJ equations (3) written in the matrix form

$$
h=a, \quad \text { with } h=\left(c, h_{n-1}, \ldots, h_{0}\right)^{T} \text { and } a=\left(c, a_{n-1}, \ldots, a_{0}\right)^{T},
$$

from the left by $w$ one gets $w h=w a$, or according to the condition (8), $f=w a$ i.e.

$$
f_{i}\left(\lambda_{i}, \frac{\partial W}{\partial \lambda_{i}}\right)=c \lambda_{i}^{n}+a_{n-1} \lambda_{i}^{n-1}+\ldots+a_{0}, \quad i=1, \ldots, n
$$


which implies the existence of the separated solution (匹) for the system of HJ equations (3). In consequence, the system (3) splits into a decoupled set of ODE's

$$
f_{i}\left(\lambda_{i}, \frac{d W_{i}}{d \lambda_{i}}\right)=c \lambda_{i}^{n}+a_{n-1} \lambda_{i}^{n-1}+\ldots+a_{0}, \quad i=1, \ldots, n .
$$

which concludes the proof.

Let us now restrict to a special case, when $f_{i}\left(\lambda_{i}, \mu_{i}\right)$ is quadratic in momenta $\mu_{i}$

$$
f_{i}\left(\lambda_{i}, \mu_{i}\right)=f_{i}\left(\lambda_{i}\right) \mu_{i}^{2}+g_{i}\left(\lambda_{i}\right),
$$

(for motivation of this choice see the end of this section) i.e. to the case when $\mathcal{M}=T^{*} Q \times \mathbf{R}$, where $Q$ is some Riemannian (pseudo-Riemannian) manifold and $T^{*} Q$ its cotangent bundle. Then the condition (8) can be put in the form

$$
\left(\begin{array}{cccc}
\lambda_{1}^{n-1} & \lambda_{1}^{n-2} & \ldots & 1 \\
\lambda_{2}^{n-1} & \lambda_{2}^{n-2} & \ldots & 1 \\
\vdots & \vdots & \ldots & \vdots \\
\lambda_{n}^{n-1} & \lambda_{n}^{n-2} & \ldots & 1
\end{array}\right)\left(\begin{array}{c}
h_{n-1} \\
h_{n-2} \\
\vdots \\
h_{0}
\end{array}\right)=\left(\begin{array}{c}
f_{1}\left(\lambda_{1}\right) \mu_{1}^{2}+g_{1}\left(\lambda_{1}\right)-\lambda_{1}^{n} c \\
f_{2}\left(\lambda_{2}\right) \mu_{2}^{2}+g_{2}\left(\lambda_{2}\right)-\lambda_{2}^{n} c \\
\vdots \\
f_{n}\left(\lambda_{n}\right) \mu_{n}^{2}+g_{n}\left(\lambda_{n}\right)-\lambda_{n}^{n} c
\end{array}\right) .
$$

This is a system of linear equations with respect to $h_{i}$. It has the solution [2]

$$
h_{i}=\sum_{k=1}^{n} G_{i}^{k k}(\lambda) \mu_{k}^{2}+V_{i}(\lambda)+c \rho_{i}(\lambda), \quad i=0, \ldots, n-1
$$

where

$$
G_{i}^{k k}(\lambda)=-\frac{\partial \rho_{i}}{\partial \lambda_{k}} \frac{f_{k}\left(\lambda_{k}\right)}{\Delta_{k}} \quad, \quad V_{i}(\lambda)=-\sum_{k=1}^{n} \frac{\partial \rho_{i}}{\partial \lambda_{k}} \frac{g_{k}\left(\lambda_{k}\right)}{\Delta_{k}}
$$

$\Delta_{i}=\prod_{j \neq i}\left(\lambda_{i}-\lambda_{j}\right)$, and $\rho_{i}(\lambda)$ are the polynomials of order $n$ in $\lambda$, defined by the relation

$$
\left(\lambda-\lambda_{1}\right)\left(\lambda-\lambda_{2}\right) \ldots\left(\lambda-\lambda_{n}\right)=\sum_{i=0}^{n} \rho_{i} \lambda^{i}
$$


(i.e. Viète polynomials). It can be shown that in the language of Riemannian geometry $G_{i}$ are second order contravariant tensors on $Q$; the tensor $G_{n-1}$ represents a contravariant metric tensor, i.e. the inverse of a standard covariant metric $g$ on $Q: G_{n-1}=g^{-1}$, while the remaining $n-1$ ones are Killing tensors. From (13) it follows that the constants of motion for geodesic case have the form

$$
\sum_{k=1}^{n} G_{i}^{k k}(\lambda) \mu_{k}^{2}=\sum_{k=1}^{n}\left(\varphi^{-1}\right)_{i}^{k} \mu_{k}^{2}
$$

where $\varphi_{i}^{k}=\lambda_{i}^{n-k} / f_{i}\left(\lambda_{i}\right)$ is a classical Stäckel matrix. It is worth mentioning here that the theory of separation of variables on Riemannian manifolds has been developed by Eisenhart [16] who used the concept of Killing tensors to analyze integrals of geodesic motion. The intrinsic formulation of Eisenhart's theory has been presented in [17] where the concept of Killing web was introduced, while the application of the theory to bi-Hamiltonian systems has been presented in 18 .

From (2) it can be shown that in $(\lambda, \mu)$ coordinates the operators $\Pi_{0}$ and $\Pi_{1}$ attain the form

$$
\begin{gathered}
\Pi_{0}=\left[\begin{array}{cc|c}
0 & I & 0 \\
-I & 0 & 0 \\
\hline * & 0
\end{array}\right] \\
\Pi_{1}=\left[\begin{array}{cc|c}
0 & \Lambda & \partial h_{n-1} / \partial \mu \\
-\Lambda & 0 & -\partial h_{n-1} / \partial \lambda \\
\hline * & 0
\end{array}\right]
\end{gathered}
$$

with the diagonal matrix $\Lambda=\operatorname{diag}\left(\lambda_{1, \ldots}, \lambda_{n}\right)$ and where the symbol $*$ denotes the elements that make the matrices skew-symmetric. In fact there exists a whole family of separated coordinates $\left(\lambda^{\prime}, \mu^{\prime}, c^{\prime}\right)$ which preserve the forms (14) and (17) of $h_{i}$ and $\Pi_{i}$ and which are related to the set $\left(\lambda, \mu, c^{\prime}\right)$ by a canonical (gauge) transformation

$$
\lambda_{i}^{\prime}= \pm \lambda_{i}, \quad \mu_{i}^{\prime}= \pm \mu_{i}+\vartheta_{i}\left(\lambda_{i}\right), \quad i=1, \ldots, n,
$$

where $\vartheta$ is an arbitrary smooth function. In the separated coordinates the Poisson pencil $\Pi_{\xi}$ and the chain (2) can be projected onto symplectic leaf $S_{c^{\prime}}$ 
of $\Pi_{0}(\operatorname{dim} S=2 n)$ producing a non-degenerated Poisson pencil on $S_{c^{\prime}}$ of the form $\theta_{\xi}=\theta_{1}-\xi \theta_{0}$, where

$$
\theta_{0}=\left[\begin{array}{cc}
0 & I \\
-I & 0
\end{array}\right], \quad \theta_{1}=\left[\begin{array}{cc}
0 & \Lambda \\
-\Lambda & 0
\end{array}\right]
$$

is a nondegenerate Poisson pencil on $S_{c^{\prime}}$. Hence, $S_{c^{\prime}}$ is a Poisson-Nijenhuis manifold where the related Nijenhuis tensor $\mathcal{N}$ and its adjoint $\mathcal{N}^{*}$

$$
\mathcal{N}=\theta_{1} \circ \theta_{0}^{-1}=\left[\begin{array}{cc}
\Lambda & 0 \\
0 & \Lambda
\end{array}\right] \quad \mathcal{N}^{*}=\theta_{0}^{-1} \circ \theta_{1}=\left[\begin{array}{cc}
\Lambda & 0 \\
0 & \Lambda
\end{array}\right]
$$

are diagonal (here we use the name of Nijenhuis tensor for a second order tensor with a vanishing Nijenhuis torsion). This motivates the following definition 19.

Definition 3 The coordinates $\left(\lambda, \mu, c^{\prime}\right)$ in which $\Pi_{0}$ and $\Pi_{1}$ have the form (17) (or, equivalently, in which the tensor $\mathcal{N}=\theta_{1} \circ \theta_{0}^{-1}$ has the diagonal form (20), are called the Darboux-Nijenhuis (DN) coordinates.

The tensors $\mathcal{N}$ and $\mathcal{N}^{*}$ are not equal, since $\mathcal{N}$ act on the space of vector fields while $\mathcal{N}^{*}$ acts on the space of one-forms on $\mathcal{M}$. Notice that $\rho_{i}(\lambda)$ are coefficients of minimal polynomial of the Nijenhuis tensor

$$
(\operatorname{det}(\lambda I-\mathcal{N}))^{1 / 2}=\operatorname{det}(\lambda I-\Lambda)=\prod_{i=1}^{n}\left(\lambda-\lambda_{i}\right)=\sum_{i=0}^{n} \rho_{i} \lambda^{i}, \quad \rho_{n}=1 .
$$

Let us now perform an arbitrary, not necessary canonical, $c^{\prime}$-independent, nondegenerate coordinate transformation $(\lambda, \mu) \rightarrow(q, p)$, preserving a quadratic dependence on momenta in all $h_{k}, k=0, \ldots, n-1$. It can be shown that after such transformation, i.e. in the variables $\left(q, p, c^{\prime}\right)$ the operators $\Pi_{0}$ and $\Pi_{1}$ will have the following form

$$
\begin{gathered}
\Pi_{0}=\left[\begin{array}{cc|c}
0 & -\widetilde{G}(q) & 0 \\
\widetilde{G}^{T}(q) & \widetilde{F}(q, p) & 0 \\
\hline * & 0
\end{array}\right] \\
\Pi_{1}=\left[\begin{array}{cc}
0 & G(q) \\
\frac{-G^{T}(q)}{*} F(q, p) & \Pi_{0} \circ d h_{n-1}
\end{array}\right]
\end{gathered}
$$


and the minimal polynomial of the Nijenhuis tensor becomes

$$
(\operatorname{det}(\xi I-\mathcal{N}))^{1 / 2}=\frac{\operatorname{det}(\xi \widetilde{G}+G)}{\operatorname{det} \widetilde{G}},
$$

Obviously, in a real situation we start from a given bi-Hamiltonian chain (2) in arbitrary coordinates $\left(q, p, c^{\prime}\right)$, derived by some method, and find the appropriate transformation to DN coordinates $\left(\lambda, \mu, c^{\prime}\right)$. In a typical situation one starts with the Hamiltonians $h_{i}$ that written in the coordinates $\left(q, p, c^{\prime}\right)$ are quadratic in momenta $p$. If the transformation to $\left(\lambda, \mu, c^{\prime}\right)$ - variables is such that $\lambda$ depends on $q$ only, then the functions $f_{i}$ must have the form (12). This is precisely the situation one encounters in the case of bi-cofactor systems. In the next sections we will apply the ideas of this section in order to separate this class of systems.

\section{Basic facts about bi-cofactor systems}

Let us consider the systems of differential equations (in the flat space $\mathbf{R}^{n}$ ) of Newton form:

$$
\ddot{q}=M(q)
$$

where $q=\left(q_{1}, \ldots, q_{n}\right)^{T}, M(q)=\left(M_{1}(q), \ldots, M_{n}(q)\right)^{T}$ where $^{T}$ denotes the transpose of a matrix, $q_{i}=q_{i}(t)$, with $t \in \mathbf{R}$ being an independent variable and where dots denote differentiation with respect to $t$, so that $\ddot{q}_{i}=d^{2} q_{i} / d t^{2}$ etc. A simple lemma below focuses our attention on those equations of type (24) that posses a quadratic in $\dot{q}$ integral of motion.

Lemma 4 Let

$$
E(q, \dot{q})=\dot{q}^{T} A(q) \dot{q}+k(q)=\sum_{i, j=1}^{n} \dot{q}_{i} A_{i j}(q) \dot{q}_{j}+k(q)
$$

where $A=A^{T}$ is a non-degenerated, $n \times n$ symmetric matrix with $q$-dependent entries. Then $E$ is an integral of motion for the system (24) (that is $\dot{E}=0$ ) if and only if the following two equations hold:

$$
\begin{gathered}
\partial_{i} A_{j k}+\partial_{j} A_{k i}+\partial_{k} A_{i j}=0 \text { for all } i, j, k=1, \ldots, n \\
2 A M+\nabla k=0
\end{gathered}
$$


Here and in what follows $\partial_{i}=\partial / \partial q_{i}$ and $\nabla k(q)=\left(\partial_{1} k, \ldots, \partial_{n} k\right)$. This lemma can easily be proved by calculating the derivative $\dot{E} \equiv d E / d t$.

The equation (25) implies that the matrix $A$ is a Killing tensor. We will restrict ourselves to a class of solutions of (25) that have the form

$$
A=\operatorname{cof}(G)
$$

with

$$
G=\alpha q q^{T}+\beta q^{T}+q \beta^{T}+\gamma
$$

where cof means the cofactor matrix (so that $\operatorname{cof}(G) G=\operatorname{det}(G)$ or, in case when $G$ is invertible, $\left.\operatorname{cof}(G)=\operatorname{det}(G) G^{-1}\right), \alpha$ is a real constant, $\beta=$ $\left(\beta_{1}, \ldots, \beta_{n}\right)^{T}$ is a column vector of constants and where $\gamma$ is a symmetric $n \times n$ constant matrix. It is easy to show that for $n=2$ it is the general solution of (25). For higher $n$ the general solution of (25) depends on $n(n+$ $1)^{2}(n+2) / 12$ parameters (its basis can be found e.g. in [20]) while (27) has only $1+n+n(n+1) / 2$ parameters and is therefore far from being a general solution. It turns out, however, that this particular solution has interesting properties that make it worth studying. It originates in a natural way when one considers a broad class of Poisson pencils of type (34) (see [12]). It leads to the notion of bi-cofactor systems that admit many differential-algebraic properties, among them interesting recursion formulas that allow to generate non-trivial bi-cofactor systems from a trivial (geodesic) flow (see below). It has been recently generalized to the case of Riemannian manifolds [21]. In this more general setting it can be demonstrated that the matrix $G$ given by (28) is a conformal Killing tensor, which leads to the orthogonal separability of geodesic Hamilton-Jacobi equation [18].

The equation (26) implies that whenever $\operatorname{det}(A) \neq 0$ the force $M$ can be written in the quasi-potential form $M=-\frac{1}{2} A^{-1} \nabla k$, which generalizes the usual potential case and which reduces to the potential case when $A=\frac{1}{2} I$, where $I$ stands for the identity matrix. Clearly, in case when our system (24) has the second - functionally independent of $E$ - integral of motion of the form

$$
\tilde{E}(q, \dot{q})=\dot{q}^{T} \tilde{A}(q) \dot{q}+\tilde{k}(q)
$$

with an invertible matrix $\tilde{A}$, then it can be written in a quasi-potential form in two distinct ways. It motivates the following definition: 
Definition 5 A system of equations

$$
\ddot{q}=M(q)=-\frac{1}{2} A^{-1}(q) \nabla k(q)=-\frac{1}{2} \tilde{A}^{-1}(q) \nabla \tilde{k}(q)
$$

where $A$ and $\tilde{A}$ are two linearly independent matrices of the cofactor form (27)-(28) :

$$
\begin{aligned}
& A=\operatorname{cof}(G), \quad G=\alpha q q^{T}+\beta q^{T}+q \beta^{T}+\gamma \\
& \tilde{A}=\operatorname{cof}(\tilde{G}), \quad G=\tilde{\alpha} q q^{T}+\tilde{\beta} q^{T}+q \tilde{\beta}^{T}+\tilde{\gamma}
\end{aligned}
$$

and where $k=k(q)$ and $\tilde{k}=\tilde{k}(q)$ are two scalar functions, is called a bicofactor system.

The bi-cofactor systems were first studied in [11, [12] and in [13], where they were called cofactor pair systems. In this article we will deal exactly with this type of systems: we will show how to perform separation of variables for quasi-potential systems of the bi-cofactor form.

An important property of such systems is that they actually admit $n$ constants of motion that are quadratic in $\dot{q}$

Theorem 6 (Hans Lundmark, 137) If the Newton system (24) has a bicofactor form (29), then it has $n$ integrals of motion of the form

$$
E_{i}(q, \dot{q})=\dot{q}^{T} A_{i}(q) \dot{q}+k_{i}(q), \quad i=0, \ldots, n-1
$$

where the matrices $A_{i}$ are defined as coefficients in the polynomial expansion of $\operatorname{cof}(G+\xi \tilde{G})$ with respect to the real parameter $\xi$ :

$$
\operatorname{cof}(G+\xi \tilde{G})=\sum_{i=0}^{n-1} A_{i} \xi^{i}
$$

with $A_{0}=\operatorname{cof}(G), A_{n-1}=\operatorname{cof}(\tilde{G})$ and where $k_{0}=k$ and $k_{n-1}=\tilde{k}$. Consequently, in case when all matrices $A_{i}$ are invertible, such system can be written in a quasi-potential form on $n$ distinct ways:

$$
\ddot{q}=M(q)=-\frac{1}{2} A_{i}^{-1} \nabla k_{i}, \quad i=0, \ldots, n-1
$$


Remark 7 In the notation as above we have of course $E_{0}=E$ and $E_{n-1}=$ $\tilde{E}$.

Of course, for a given pair of matrices $G$ and $\tilde{G}$ not every function $k$ will have a counterpart $\tilde{k}$ that will satisfy the equation (29). However, there exists a recursion formula that from a given bi-cofactor system produces a new bi-cofactor system.

Proposition 8 Let $\ddot{q}=M(q)$ be a bi-cofactor system of the form (29) with the integrals given by (30). Let also $k_{\xi}=\sum_{i=0}^{n-1} k_{i} \xi^{i}$ (with $k_{n-1}=\breve{k}$ ). Then the functions $l_{i}, i=0, \ldots, n-1$ defined as $l_{\xi}=\sum_{i=0}^{n-1} l_{i} \xi^{i}$ through

$$
l_{\xi}=\frac{\operatorname{det}(G+\xi \tilde{G})}{\operatorname{det}(\tilde{G})} \tilde{k}-\xi k_{\xi}
$$

satisfy the relation $A_{i}^{-1} \nabla l_{i}=A_{j}^{-1} \nabla l_{j}$ for all $i, j=0, \ldots, n-1$ and are in consequence right hand sides of a new bi-cofactor system of the form (31) (with $l_{i}$ instead of $k_{i}$ ).

The proof of this statement can be found in 13]. This formula makes it possible to produce infinite sequences of bi-cofactor systems starting for example from a simple geodesic equation $\ddot{q}=0$ which is obviously of a bicofactor form with for example $k_{0}=0$ and $k_{n-1}=1$. Also, it can easily be inverted in order to express old quasi-potentials $k_{i}$ through the new quasipotentials $l_{i}$ :

$$
k_{\xi}=\frac{1}{\xi}\left(\frac{\operatorname{det}(G+\xi \tilde{G})}{\operatorname{det}(G)} l-l_{\xi}\right)
$$

In consequence, by using (33) it is possible to produce "lower" systems from the "higher" ones, and if we start from the geodesic equation $\ddot{q}=0$, we obtain in general the family of "negative" systems different from the sequence of systems obtained from $\ddot{q}=0$ by the use of (32).

It has been proved [12], [13] that the system (29) can be embedded in a bi-Hamiltonian system. In order to make this statement more precise, let us consider a following skew-symmetric operator pencil associated with the system (29): 


$$
\begin{aligned}
\Pi_{\xi}=\Pi_{1}-\xi \Pi_{0} \equiv\left[\begin{array}{cc|c}
0 & G(q) & p \\
-G(q) & F(q, p) & M(q)+2 c N(q) \\
\hline * & 0
\end{array}\right] \\
-\xi\left[\begin{array}{cc|c}
0 & -\tilde{G}(q) & 0 \\
\tilde{G}(q) & -\tilde{F}(q, p) & -2 c \tilde{N}(q) \\
\hline * & 0
\end{array}\right]
\end{aligned}
$$

where both $\Pi_{1}$ and $\Pi_{0}$ are $(2 n+1) \times(2 n+1)$ matrices/operators acting in the $(2 n+1)$-dimensional space $\mathcal{M}=\mathbf{R}^{2 n+1}$ with Cartesian coordinates labelled with $(q, p, c)$ where $q=\left(q_{1}, \ldots, q_{n}\right)^{T}, p=\left(p_{1}, \ldots, p_{n}\right)^{T}, c \in \mathbf{R}$. The $n \times n$ symmetric matrices $G$ and $\tilde{G}$ are exactly the matrices that define our system (29). The $n \times 1$ matrices $N$ and $\tilde{N}$ are given by

$$
N=\alpha q+\beta, \quad \tilde{N}=\tilde{\alpha} q+\tilde{\beta} .
$$

The $n \times n$ matrices $F$ and $\tilde{F}$ are defined by

$$
F=N p^{T}-p N^{T}, \quad \tilde{F}=\tilde{N} p^{T}-p \tilde{N}^{T}
$$

As usual, the asterisk $*$ denotes the elements that makes our matrices skewsymmetric. This operator pencil is a Poisson pencil precisely due to the fact, that the term $M(q)$ can be represented as $M(q)=-\frac{1}{2} A_{i}^{-1} \nabla k_{i}$ for all $i=0, \ldots n-1$. One should also notice that this pencil is linear in the variable $c$ and that it has a maximal rank $2 n$. Thus, according to Section 2 , the Casimir function $h_{\xi}$ (see Definition 1); obviously, in our variables $d=$ $\left.(\partial / \partial q, \partial / \partial p, \partial / \partial c)^{T}\right)$ of our Poisson operator $\Pi_{\xi}$ is a polynomial of $\operatorname{grad} n$ in $\xi$. In fact, the Casimir $h_{\xi}$ of $\Pi_{\xi}$ has the form:

$$
h_{\xi}(q, p, c)=p^{T} \operatorname{cof}(G+\xi \tilde{G}) p+k_{\xi}(q)-2 c \operatorname{det}(G+\xi \tilde{G})
$$

where $k_{\xi}(q)=\sum_{i=0}^{n-1} k_{i}(q) \xi^{i}$ are as in Proposition 8 above. Thus, $h_{\xi}=$ $\sum_{i=0}^{n} h_{i} \xi^{i}$ with the functions $h_{i}(q, p, c)$ given explicitly by

$$
\begin{gathered}
h_{i}(q, p, c)=E_{i}(q, p)-2 c D_{i}, \quad i=0, \ldots, n-1 \\
h_{n}(q, c)=-2 c D_{n}
\end{gathered}
$$


with $D_{i}=D_{i}(q)$ defined as

$$
\sum_{i=0}^{n} D_{i}(q) \xi^{i}=\operatorname{det}(G+\xi \tilde{G})
$$

so that $D_{0}=\operatorname{det}(G)$ and $D_{n}=\operatorname{det}(\tilde{G})$. The functions $E_{i}$ in (36) are just the constants of motion (30) of our system (29). By expanding the equation $\Pi_{\xi} \circ d h_{\xi}=0$ in powers of $\xi$ and comparing the coefficients at equal powers we obtain the bi-Hamiltonian chain of the form (2) which by theorem of Magri 222 is completely integrable in the sense of Liouville. This means that the evolutionary equations

$$
\frac{d}{d t}\left[\begin{array}{l}
q \\
p \\
c
\end{array}\right]=\Pi_{1} \circ d h_{i}=\Pi_{0} \circ d h_{i-1}, i=1, \ldots, n
$$

associated with (2) are Liouville-integrable.

Let us now investigate the relation of our chain (2) (or of our Poisson pencil (34)) with the system (29). One can show, that the last equation in (37) has at the hyperplane $c=0$ the following form:

$$
\frac{d}{d t}\left[\begin{array}{c}
q \\
p \\
c
\end{array}\right]=-2 \operatorname{det}(\tilde{G})\left[\begin{array}{c}
p \\
M \\
0
\end{array}\right]
$$

so that the hyperplane $c=0$ is invariant with respect to this equation. On the other hand, if we set $\dot{q}=p$ in $(\sqrt[29]{)}$, we obtain its equivalent form

$$
\frac{d}{d t}\left[\begin{array}{l}
q \\
p
\end{array}\right]=\left[\begin{array}{l}
p \\
M
\end{array}\right]
$$

which differ from $(38)$ only by the coefficient $-2 \operatorname{det}(\tilde{G})$. It means that both systems have the same trajectories in the $(q, p)$-space, although traversed at different speed.

Lemma 9 (rescaling) Let $x(t)=\left(x_{1}(t), \ldots, x_{n}(t)\right)$ be a solution of a first order differential equation $\dot{x}=X(x)$ in $\mathbf{R}^{n}$ with $x(0)=x_{0} \in \mathbf{R}^{n}$ and let $\alpha: \mathbf{R}^{n} \rightarrow \mathbf{R}$ be a scalar function. Then the function $y(t)=x\left(\tau^{-1}(t)\right)$ with $\tau(t)=\int \frac{1}{\alpha(x(t))} d t$ (chosen so that $\tau(0)=0$ ) solves the differential equation $\dot{y}=\alpha(y) X(y)$ with the initial condition $y(0)=x_{0}$. 
The proof of this lemma is elementary. This lemma implies that knowing a particular solution of (38) we can write down the corresponding solution of (39), just by identifying $\alpha$ with $-1 /(2 \operatorname{det}(\tilde{G}))$. In the next section we will show how one can solve all the equations (37) (and thus also the bi-cofactor system (39)) by a procedure that separates variables in Hamilton-Jacobi equations that correspond to all Hamiltonians $h_{i}$ of the chain (2).

More information about bi-cofactor systems as well as more detailed explanations of the facts mentioned above can be found in [12, [13, ,15. In [21 and in [23] one can find a generalization of many of the above mentioned statements to the case of Riemannian manifolds.

\section{Separation of variables}

In the preceding section we explained how our bi-cofactor system (29) can be embedded in a bi-Hamiltonian chain (2). The main goal of this article is to present the procedure that leads to separation of variables in the HamiltonJacobi equations corresponding to the Hamiltonians $h_{i}$. According to the rescaling lemma above, such a procedure will yield a solution of our bicofactor system as well. This procedure is based on the results obtained in [8], [9], [24] and other papers.

Let us begin by adjusting our coordinate system so that one of the new coordinates correspond to the foliation of $\mathcal{M}$ into symplectic leaves of $\Pi_{0}$. We will obtain it by rescaling $c$. Let us thus introduce the following curvilinear coordinate system in $\mathcal{M}$.

$$
q_{i}^{\prime}=q_{i}, p_{i}^{\prime}=p_{i}, i=1, \ldots, n, \quad c^{\prime}=h_{n}(q, c)=-2 c \operatorname{det}(\tilde{G})
$$

(observe that the hypersurfaces $c=0$ and $c^{\prime}=0$ coincide). One should notice that this transformation does not depend on a particular choice of $k$ and $\tilde{k}$ (since the operator $\Pi_{0}$ does not depend on $k$ and $\tilde{k}$ ) but only on the choice of $\tilde{G}$. In what follows we will write $q_{i}$ and $p_{i}$ instead of $q_{i}^{\prime}$ and $p_{i}^{\prime}$. In the $q, p, c^{\prime}$-variables the chain (2) has the same form; however, the explicit form of operators $\Pi_{0}$ and $\Pi_{1}$ changes to:

$$
\Pi_{0}=\left[\begin{array}{cc|c}
0 & -\tilde{G} & 0 \\
\tilde{G} & -\tilde{F} & 0 \\
\hline * & 0
\end{array}\right]
$$




$$
\Pi_{1}=\left[\begin{array}{cc|c}
0 & G & -2 p \operatorname{det}(\tilde{G}) \\
-G & F & 2 c^{\prime}\left(N-G \tilde{G}^{-1} \tilde{N}\right)-2 \operatorname{det}(\tilde{G}) M \\
\hline * & 0
\end{array}\right]
$$

(cf (22)), while the Hamiltonians $h_{i}$ attain the form:

$$
\begin{aligned}
& h_{i}\left(q, p, c^{\prime}\right)=E_{i}(q, p)+c^{\prime} \frac{D_{i}}{D_{n}}, \quad i=0, \ldots, n-1 \\
& h_{n}=c^{\prime}
\end{aligned}
$$

In the new variables the symplectic leaves of $\Pi_{0}$ have the desired form $c^{\prime}=$ const. Naturally, the question arises, if we could not choose a different pencil $\Pi$ so that the formula (38) would not contain the factor $-2 \operatorname{det}(\tilde{G})$, which would make Lemma 9 unnecessary, but we were not able to do so yet.

We are now in position to present the main theorem of this article.

Theorem 10 If the roots $\lambda_{i}=\lambda_{i}(q)$ of the equation

$$
\operatorname{det}(G+\lambda \tilde{G})=0
$$

are functionally independent, then in the variables $\lambda=\left(\lambda_{1}, \ldots \lambda_{n}\right)^{T}, \mu=$ $\left(\mu_{1}, \ldots \mu_{n}\right)^{T}, c^{\prime}$ given by

$$
\lambda_{i}(q) \text { as roots of (49) }
$$

$$
\mu_{i}(q, p)=-\frac{1}{2} \frac{\Omega^{T} \operatorname{cof}\left(G+\lambda_{i}(q) \tilde{G}\right) p}{\Omega^{T} \operatorname{cof}\left(G+\lambda_{i}(q) \tilde{G}\right) \Omega} \quad i=1, \ldots, n
$$

where $\Omega=G \tilde{G}^{-1} \tilde{N}-N$, the operators $\Pi_{0}$ and $\Pi_{1}$ attain the form

$$
\begin{gathered}
\Pi_{0}=\left[\begin{array}{cc|c}
0 & I & 0 \\
-I & 0 & 0 \\
\hline * & 0
\end{array}\right] \\
\Pi_{1}=\left[\begin{array}{cc|c}
0 & \Lambda & \partial h_{n-1} / \partial \mu \\
-\Lambda & 0 & -\partial h_{n-1} / \partial \lambda \\
\hline * & 0
\end{array}\right]
\end{gathered}
$$


(cf (29)), with the diagonal matrix $\Lambda=\operatorname{diag}\left(\lambda_{1, \ldots}, \lambda_{n}\right)$, while the Hamiltonians $h_{i}$ have the form

$$
\begin{aligned}
& h_{i}\left(\lambda, \mu, c^{\prime}\right)=-\sum_{k=1}^{n} \frac{\partial \rho_{i}}{\partial \lambda_{k}} \frac{f_{k}\left(\lambda_{k}, \mu_{k}\right)}{\Delta_{k}}+c^{\prime} \rho_{i}(\lambda), \quad i=0, \ldots, n \quad(n \geq 2) \\
& h_{1}\left(\lambda, \mu, c^{\prime}\right)=f(\lambda, \mu)+c^{\prime} \lambda, \quad(n=1)
\end{aligned}
$$

(cf (14)), with some functions $f_{k}$ depending only on one pair $\lambda_{k}, \mu_{k}$ of the variables, $\Delta_{k}=\prod_{j \neq k}\left(\lambda_{k}-\lambda_{j}\right)$, and where $\rho_{i}(\lambda)$ are the Viète polynomials (15). Moreover, in the variables $\lambda, \mu, c^{\prime}$ the recursion formula (39) attains the form

$$
l_{\xi}(\lambda)=\operatorname{det}(\xi I-\Lambda) \tilde{k}(\lambda)-\xi k_{\xi}(\lambda)
$$

i.e. the formula (39) is invariant with respect to the change of variables $q \rightarrow \lambda$.

Remark 11 It is worth mentioning that the coordinates $\lambda(q)$ defined by (41) are in general not orthogonal, and that the gradients $\nabla \lambda_{i}(q)$ are eigenvectors of the matrix $G \tilde{G}^{-1}$, i.e. $\left(G+\lambda_{i} \tilde{G}\right) \nabla \lambda_{i}=0$. Moreover, $\nabla \lambda_{i}(q)$ are $\tilde{G}$ orthogonal: $\nabla \lambda_{i} G \nabla \lambda_{j}=0$ for $i \neq j$. In case when one of the matrices, say $\tilde{G}$, is equal to the identity matrix (that is when our system (29) becomes potential) the above transformation (41)-(49) reduces to the classical formula for point transformation to separation coordinates for natural Hamiltonian systems.

Remark 12 The terms $D_{i} / D_{n}$ in the Hamiltonians $h_{i}$ attain the form $\rho_{i}(\lambda)$ in (44) precisely due to the fact that $D_{i} / D_{n}$ are coefficients in the polynomial expansion of $\operatorname{det}(G+\lambda \tilde{G}) / \operatorname{det}(\tilde{G})=\sum_{i=0}^{n} \rho_{i} \lambda^{i} \quad(c f$. (2马)).

Remark 13 The formulae (49)-(压) provide us with a transformation that is independent of the particular choice of the functions $k$ and $\tilde{k}$ in the bicofactor system (29), i.e. this transformation will simultaneously separate all the bi-cofactor systems with the same matrices $G$ and $\tilde{G}$.

Theorem 10 means that the coordinates $\left(\lambda, \mu, c^{\prime}\right)$ are Darboux-Nijenhuis coordinates for our operators $\Pi_{0}, \Pi_{1}$. According to results of Section 2, we have 
Corollary 14 The Hamilton-Jacobi equations for the Hamiltonians $h_{i}\left(\lambda, \mu, c^{\prime}\right)$

$$
h_{i}\left(\lambda, \frac{\partial W}{\partial \lambda}, c^{\prime}\right)=a_{i} \quad i=0,1, \ldots, n
$$

where $W(\lambda, a)$ is a generating function for the transformation $(\lambda, \mu) \rightarrow(b, a)$, separate under the ansatz $W(\lambda, a)=\sum_{i=1}^{n} W_{i}\left(\lambda_{i}, a\right)$ into system of ODE's of the form

$$
f_{k}\left(\lambda_{k}, \frac{d W_{k}}{d \lambda_{k}}\right)=c^{\prime} \lambda_{k}^{n}+a_{1} \lambda_{k}^{n-1}+\cdots+a_{n}
$$

Proof. The Hamilton-Jacobi equations for Hamiltonians (44) can be treated as a system of $n$ linear equations for functions $f_{i}$. Applying the Cramer rule to this system we arrive at (46).

Thus, we are able to find $W$ up to quadratures. If we denote the evolution parameter associated with $h_{j}$ by $t_{j}$, then in the new variables $(b, a)$ defined implicitly as usual:

$$
b_{i}=\frac{\partial W(\lambda, a)}{\partial a_{i}}, \quad \mu_{i}=\frac{\partial W(\lambda, a)}{\partial \lambda_{i}} \quad i=1, \ldots, n
$$

the flow associated with $h_{j}$ has the trivial form (5) so that the transformation $(\lambda, \mu) \rightarrow(b, a)$ simultaneously trivializes Hamilton equations generated by all the Hamiltonians $h_{i}$.

We will now sketch the proof of Theorem 10. Consider a symplectic leaf $S_{c^{\prime}}=\left\{\left(q, p, c^{\prime}\right): c^{\prime}=\right.$ const $\}$ of $\Pi_{0}$. Let us choose a vector field transversal to the symplectic foliation of $\Pi_{0}$ as $Z=\frac{\partial}{\partial c^{\prime}}$. It can be shown by direct calculation that

$$
L_{Z} \Pi_{0}=0, \quad L_{Z} \Pi_{1}=X \wedge Z \quad \text { with } X=\Pi_{0} \circ d\left(Z\left(h_{n-1}\right)\right)
$$

where $L_{Z}$ is a Lie derivative operator in the direction of the vector field $Z$. The above relations guarantee that we can perform a projection of both $\Pi_{0}$ and $\Pi_{1}$ onto the symplectic leaf $S_{c^{\prime}}$ of $\Pi_{0}$. The obtained $2 n$-dimensional Poisson operators $\theta_{0}$ and $\theta_{1}$ have the form:

$$
\theta_{0}(q, p)=\left[\begin{array}{cc}
0 & -\tilde{G}(q) \\
\tilde{G}(q) & -\tilde{F}(q, p)
\end{array}\right] \quad \theta_{1}(q, p) \equiv\left[\begin{array}{cc}
0 & G(q) \\
-G(q) & F(q, p)
\end{array}\right]
$$


The corresponding Nijenhuis tensor $\mathcal{N}=\theta_{1} \circ \theta_{0}^{-1}$ has the minimal polynomial of the form $\operatorname{det}(G+\lambda \tilde{G}) / \operatorname{det}(\tilde{G})(\operatorname{cf}(23))$ and its roots are precisely the roots of (40). On the other hand, the roots of the minimal polynomial of $\mathcal{N}$ define - according to (21) - the first half of the transformation $(q, p) \rightarrow(\lambda, \mu)$ to DN coordinates in which the operators $\theta_{0}$ and $\theta_{1}$ have the form (19). Due to the last but one equation in the chain (2) this implies that in the DN coordinates the operators $\Pi_{0}$ and $\Pi_{1}$ must have the form $(43)$.

We will now show that the remaining part of the transformation $(q, p) \rightarrow$ $(\lambda, \mu)$ to the DN coordinates, i.e. the expression for $\mu=\mu(q, p)$, must be of the form (42). We will do it in few steps.

Lemma 15 In the DN coordinates the vector field $X$ has a simple form:

$$
X=\sum_{i=1}^{n} \frac{\partial}{\partial \mu_{i}}
$$

Proof. It is enough to calculate $X=\Pi_{0} \circ d\left(Z\left(h_{n-1}\right)\right)$ in the DNcoordinates:

$$
d\left(Z\left(h_{n-1}\right)\right)=d\left(\frac{\partial}{\partial c^{\prime}}\left(h_{n-1}\right)\right)=d\left(\frac{D_{n-1}}{D_{n}}\right)=-d\left(\lambda_{1}+\cdots+\lambda_{n}\right)
$$

where the last equality is due to the fact that $D_{n-1} / D_{n}$ is precisely the same term that the last but one in the polynomial expansion of $\operatorname{det}(G+\lambda \tilde{G}) / \operatorname{det}(\tilde{G})$ that in turn is precisely the Viète polynomial $\rho_{n-1}=-\left(\lambda_{1}+\cdots+\lambda_{n}\right)$. Thus,

$$
\Pi_{0} \circ d\left(Z\left(h_{n-1}\right)\right)=-\Pi_{0} \circ d\left(\lambda_{1}+\cdots+\lambda_{n}\right)=\sum_{i=1}^{n} \frac{\partial}{\partial \mu_{i}}
$$

Lemma 16 The function

$$
H_{i}\left(\lambda, \mu, c^{\prime}\right) \equiv \sum_{k=0}^{n} h_{k}\left(\lambda, \mu, c^{\prime}\right) \lambda_{i}^{k}
$$

(i.e. Casimir (35) written in $\left(\lambda, \mu, c^{\prime}\right)$ variables and evaluated at $\left.\lambda_{i}\right)$ depends only on the $i$-th pair $\lambda_{i}, \mu_{i}$ of the variables $\lambda, \mu$ i.e.

$$
H_{i}\left(\lambda, \mu, c^{\prime}\right)=f_{i}\left(\lambda_{i}, \mu_{i}\right)
$$

for some function $f_{i}\left(\lambda_{i}, \mu_{i}\right)$. 
Proof. We have that $\partial H_{i} / \partial c^{\prime}=\sum_{k=0}^{n} \lambda_{i}^{k} \partial h_{k} / \partial c^{\prime}=\sum_{k=0}^{n} \lambda_{i}^{k} D_{k} / D_{n}=$ $\operatorname{det}\left(G+\lambda_{i} \tilde{G}\right) / \operatorname{det}(\tilde{G})=0$ due to (40). For $j \neq i$ we observe that $\partial H_{i} / \partial \lambda_{j}=$ $\frac{\partial h_{0}}{\partial \lambda_{j}}+\lambda_{i} \frac{\partial h_{1}}{\partial \lambda_{j}}+\lambda_{i}^{2} \frac{\partial h_{2}}{\partial \lambda_{j}}+\ldots+\lambda_{i}^{n} \frac{\partial h_{n}}{\partial \lambda_{j}}, \quad i, j=1, \ldots, n$. On the other hand, due to (43) and (2)

$$
-\lambda_{j} \frac{\partial h_{k}}{\partial \lambda_{j}}-\frac{\partial h_{n-1}}{\partial \lambda_{j}} \frac{\partial h_{k}}{\partial c^{\prime}}=-\frac{\partial h_{k-1}}{\partial \lambda_{j}}, \quad k, j=1, \ldots, n
$$

so that

$$
\frac{\partial h_{k}}{\partial \lambda_{j}}=-\frac{\partial h_{n-1}}{\partial \lambda_{j}}\left(\frac{\rho_{k}}{\lambda_{j}}+\frac{\rho_{k-1}}{\lambda_{j}^{2}}+\cdots+\frac{\rho_{0}}{\lambda_{j}^{k+1}}\right)
$$

which substituted in the above expression for $\partial H_{i} / \partial \lambda_{j}$ yields zero. In a similar way one can prove that $\partial H_{i} / \partial \mu_{j}=0$ for $i \neq j$.

By Theorem 2 of Section 2 (and the pages that follow this theorem) this lemma means that our coordinates $\lambda, \mu$ indeed are separation coordinates for our systems. We will however continue our line of proof of Theorem 10.

Corollary 17 From the above lemma it follows, by Cramer rule, that $h_{i}$ must have the form (44).

Lemma 18 [24 Let $X=\Pi_{0} d\left(Z\left(h_{n-1}\right)\right)$. Suppose that $X^{r}\left(H_{i}\right)=0$ for some $r=2,3, \ldots$ and that $X^{k}\left(H_{i}\right) \neq 0$ for $k=1, \ldots, r-1$. Then

$$
\mu_{i}=\frac{X^{r-2}\left(H_{i}\right)}{X^{r-1}\left(H_{i}\right)}
$$

In order to prove this lemma it is sufficient to integrate the relation $X^{r}\left(H_{i}\right)=0$ twice, using the fact that $X=\sum_{i=1}^{n} \frac{\partial}{\partial \mu_{i}}$ and Lemma 16 and use gauge invariance of DN-coordinates in order to kill integration functions that appear after second integration.

In our case the exponent $r$ that "kills" $H_{i}$ is equal to 3 , since in our old coordinates $\left(q, p, c^{\prime}\right)$ the vector field $X$ has the form $X=\sum_{i}(\ldots) \frac{\partial}{\partial p_{i}}$ and since $h_{i}$ are quadratic in $p$. Explicit calculation of expression (47) for $r=3$ and in $q, p, c^{\prime}$-variables yields exactly (42).

The recursion formula (45) is obtained by inserting $I$ and $\Lambda$ as $-\tilde{G}$ and $G$ in (32). This concludes the proof of Theorem 10. 


\section{Examples}

We will now illustrate the content of the presented theory with examples. It is worth to note that the general theory does not provide us with any tools for calculating the functions $f_{i}$ in (44) (or in (46)). Instead, we have to calculate these functions each time we perform the variable separation of a given bi-cofactor system. In case of bi-cofactor systems however it turns out that the functions $f_{i}$ always have the form (12).

As a first example, let us consider the family of parabolic separable potentials introduced in [25]. They have the form:

$$
V^{(r)}\left(q_{1}, q_{2}\right)=\sum_{k=0}^{[r / 2]} 2^{r-2 k}\left(\begin{array}{c}
r-k \\
k
\end{array}\right) q_{1}^{2 k} q_{2}^{r-2 k}
$$

with the other integral of motion given by

$$
E^{(r)}=-q_{2} \dot{q}_{1}^{2}+q_{1} \dot{q}_{1} \dot{q}_{2}+q_{1}^{2} V^{(r-1)}
$$

We easily find the corresponding matrices $G$ and $\tilde{G}$

$$
G=\left[\begin{array}{cc}
0 & -q_{1} / 2 \\
-q_{1} / 2 & -q_{2}
\end{array}\right] \quad, \quad \tilde{G}=\frac{1}{2} I
$$

The recursion formula (32), applied to the geodesic equation $\ddot{q}=0$ with $k_{\xi}=\xi$ (i.e. with $k_{0}=0, k_{1}=1$ ) produces an infinite family of pairs of quasi-potentials $k_{0}^{(r)}, k_{1}^{(r)}, r=0,1, \ldots$ (with $k_{0}^{(0)}=k_{0}, k_{1}^{(0)}=k_{1}$ ) such that $\left.(\operatorname{cof}(G))^{-1} \nabla k_{0}^{(r)}=\operatorname{cof}(\tilde{G})\right)^{-1} \nabla k_{1}^{(r)} \equiv 2 \nabla k_{1}^{(r)}$. The first few are:

$$
\begin{gathered}
k_{0}^{(1)}=-q_{1}^{2}, \quad k_{1}^{(1)}=-2 q_{2} \\
k_{0}^{(2)}=2 q_{2} q_{1}^{2}, \quad k_{1}^{(2)}=q_{1}^{2}+4 q_{2}^{2} \\
k_{0}^{(3)}=-q_{1}^{4}-4 q_{1}^{2} q_{2}^{2}, \quad k_{1}^{(3)}=-4 q_{1}^{2} q_{2}-8 q_{2}^{3}
\end{gathered}
$$

where $k_{1}^{(r)}$ correspond to the potentials $V^{(r)}$ up to a factor $(-1)^{r}$ and where according to (48) $k_{0}^{(r)}=-q_{1}^{2} k_{1}^{(r-1)}$. The corresponding potential-cofactor systems $\ddot{q}=M^{(r)}(q)=-\frac{1}{2}(\operatorname{cof}(G))^{-1} \nabla k_{0}^{(r)}=-\nabla k_{1}^{(r)}$ have the form:

$$
\begin{gathered}
\ddot{q}=M^{(1)}(q)=(0,2)^{T} \\
\ddot{q}=M^{(2)}(q)=\left(-2 q_{1},-8 q_{2}\right)^{T} \\
\ddot{q}=M^{(3)}(q)=\left(8 q_{1} q_{2}, 4 q_{1}^{2}+24 q_{2}^{2}\right)^{T}
\end{gathered}
$$


so that the third one is already non-trivial. Similarly, by applying the formula (33), we can produce from the geodesic equation $\ddot{q}=0$ with $k_{\xi}=1$ (i.e. with $\left.k_{0}^{(0)}=1, k_{1}^{(0)}=0\right)$ the "negative" quasi-potentials: The first few of them are of the form

$$
\begin{gathered}
k_{0}^{(-1)}=2 q_{2} / q_{1}^{2}, k_{1}^{(-1)}=-1 / q_{1}^{2} \\
k_{0}^{(-2)}=\left(4 q_{2}^{2}+q_{1}^{2}\right) / q_{1}^{4}, k_{1}^{(-2)}=-2 q_{2} / q_{1}^{4} \\
k_{0}^{(-3)}=4 q_{2}\left(2 q_{2}^{2}+q_{1}^{2}\right) / q_{1}^{6}, \quad k_{1}^{(-3)}=-\left(4 q_{2}^{2}+q_{1}^{2}\right) / q_{1}^{6}
\end{gathered}
$$

and correspond to potential-cofactor systems $\ddot{q}=M^{(r)}(q)=-\frac{1}{2}(\operatorname{cof}(G))^{-1} \nabla k_{0}^{(r)}=$ $-\nabla k_{1}^{(r)}$ with

$$
\begin{gathered}
\ddot{q}=M^{(-1)}(q)=\left(-2 / q_{1}^{3}, 0\right)^{T} \\
\ddot{q}=M^{(-2)}(q)=\left(-8 q_{2} / q_{1}^{5}, 2 / q_{1}^{4}\right)^{T} \\
\ddot{q}=M^{(-3)}(q)=\left(-4\left(6 q_{2}^{2}+q_{1}^{2}\right) / q_{1}^{7}, 8 q_{2} / q_{1}^{6}\right)^{T}
\end{gathered}
$$

In order to check what variables will separate these systems, we have to solve the equation (40) with $G$ and $\tilde{G}$ given as in (49). An easy computation yields

$$
\lambda_{1}(q)=q_{2}-\sqrt{q_{1}^{2}+q_{2}^{2}}, \lambda_{2}(q)=q_{2}+\sqrt{q_{1}^{2}+q_{2}^{2}}
$$

The formula (42) in this case reads

$$
\mu_{i}=\frac{\sqrt{-\lambda_{1}(q) \lambda_{2}(q)}}{\lambda_{i}(q)} p_{1}+p_{2}, \quad i=1,2
$$

and it is immediate to show that the above formulas present the classical point transformation to the parabolic coordinates. Thus, not only the potentials $V^{(r)}$ but even the corresponding chain (2) is separable in the parabolic coordinates which is perhaps what we should expect. After some algebraic manipulations, the above formulas can be inverted to

$$
q_{1}=\sqrt{-\lambda_{1} \lambda_{2}} \quad, \quad q_{2}=\frac{1}{2}\left(\lambda_{1}+\lambda_{2}\right)
$$




$$
p_{1}=\frac{\sqrt{-\lambda_{1} \lambda_{2}}\left(\mu_{1}-\mu_{2}\right)}{\lambda_{1}-\lambda_{2}} \quad, \quad p_{2}=\frac{\mu_{1} \lambda_{1}-\mu_{2} \lambda_{2}}{\lambda_{1}-\lambda_{2}}
$$

which makes it possible to express the Hamiltonians $h_{i}$ in the DN coordinates $\left(\lambda, \mu, c^{\prime}\right)$. According to (36) and (30) in the old variables $\left(q, p, c^{\prime}\right)$ they have the form

$$
h_{i}=\gamma_{i}(q, p)+k_{i}(q)+c^{\prime} \frac{D_{i}}{D_{n}}
$$

where $\gamma_{i}(q, p)=p^{T} A_{i}(q) p$ is the geodesic part (geodesic Hamiltonian) of $h_{i}$. Terms $\frac{D_{i}}{D_{n}}=\rho_{i}$ have in the DN coordinates the form of Viète polynomials (15) while the form of $k_{i}$ for a given bi-cofactor system obtained by recursion (32) can easily be established either by substituting the above expressions for $q_{i}(\lambda)$ and $p_{i}(\lambda, \mu)$ in (50)-(51) or from the recursion relation (45). The result is

$$
\begin{gathered}
k_{0}^{(-3)}=\frac{-\left(\lambda_{1}+\lambda_{2}\right)\left(\lambda_{1}^{2}+\lambda_{2}^{2}\right)}{\lambda_{1}^{3} \lambda_{2}^{3}}, \quad k_{1}^{(-3)}=\frac{\lambda_{1}^{2}+\lambda_{1} \lambda_{2}+\lambda_{2}^{2}}{\lambda_{1}^{3} \lambda_{2}^{3}} \\
k_{0}^{(-2)}=\frac{\lambda_{1}^{2}+\lambda_{1} \lambda_{2}+\lambda_{2}^{2}}{\lambda_{1}^{2} \lambda_{2}^{2}}, \quad k_{1}^{(-2)}=\frac{-\left(\lambda_{1}+\lambda_{2}\right)}{\lambda_{1}^{2} \lambda_{2}^{2}} \\
k_{0}^{(-1)}=\frac{-\left(\lambda_{1}+\lambda_{2}\right)}{\lambda_{1} \lambda_{2}}, k_{1}^{(-1)}=\frac{-1}{\lambda_{1} \lambda_{2}} \\
k_{0}^{(1)}=\lambda_{1} \lambda_{2}, \quad k_{1}^{(1)}=-\left(\lambda_{1}+\lambda_{2}\right) \\
k_{0}^{(2)}=-\left(\lambda_{1}+\lambda_{2}\right) \lambda_{1} \lambda_{2} \quad, \quad k_{1}^{(2)}=\lambda_{1}^{2}+\lambda_{1} \lambda_{2}+\lambda_{2}^{2} \\
k_{0}^{(3)}=\lambda_{1} \lambda_{2}\left(\lambda_{1}^{2}+\lambda_{1} \lambda_{2}+\lambda_{2}^{2}\right), \quad k_{1}^{(3)}=-\left(\lambda_{1}^{3}+\lambda_{1}^{2} \lambda_{2}+\lambda_{1} \lambda_{2}^{2}+\lambda_{2}^{3}\right)
\end{gathered}
$$

The geodesic Hamiltonians $\gamma_{i}$ have in our case the form

$$
\begin{aligned}
& \gamma_{0}=\frac{-\lambda_{2}}{\lambda_{1}-\lambda_{2}} \frac{1}{2} \lambda_{1} \mu_{1}^{2}+\frac{-\lambda_{1}}{\lambda_{2}-\lambda_{1}} \frac{1}{2} \lambda_{2} \mu_{2}^{2} \\
& \gamma_{1}=\frac{1}{\lambda_{1}-\lambda_{2}} \frac{1}{2} \lambda_{1} \mu_{1}^{2}+\frac{1}{\lambda_{2}-\lambda_{1}} \frac{1}{2} \lambda_{2} \mu_{2}^{2}
\end{aligned}
$$


so that they indeed have the form (44) with $f_{i}$ of the form (12) (as it has been pointed out at the end of section 2) and we can identify the functions $f_{i}\left(\lambda_{i}\right)$ in (12) as $f_{i}\left(\lambda_{i}\right)=\frac{1}{2} \lambda_{i}$ which is the form that can be used in order to solve the inverse Jacobi problem associated with equations (46) and in consequence to separate the system (29).

As a second example we will consider a quite generic (but still twodimensional) bi-cofactor system with matrices $G$ and $\tilde{G}$ of the form

$$
G=\left[\begin{array}{cc}
q_{1}^{2}+1 & q_{1} q_{2} \\
q_{1} q_{2} & q_{2}^{2}
\end{array}\right] \quad, \quad \tilde{G}=\left[\begin{array}{cc}
1 & q_{1} \\
q_{1} & 2 q_{2}
\end{array}\right]
$$

It is no longer potential. The first few of the bi-cofactor systems produced by the recursion formulae (32) and (33) are defined by the quasipotentials

$$
\begin{gathered}
k_{0}^{(-2)}=\frac{4+2 q_{2}+2 q_{2}^{2}+q_{1}^{2}}{q_{2}^{2}}, k_{1}^{(-2)}=\frac{-\left(2 q_{1}^{2}-2 q_{2}^{2}+q_{1}^{2} q_{2}-4 q_{2}\right)}{q_{2}^{3}} \\
k_{0}^{(-1)}=\frac{2+q_{2}}{q_{2}}, k_{1}^{(-1)}=\frac{2 q_{2}-q_{1}^{2}}{q_{2}^{2}} \\
k_{0}^{(1)}=\frac{q_{2}^{2}}{2 q_{2}-q_{1}^{2}} \quad, \quad k_{1}^{(1)}=\frac{q_{2}\left(2+q_{2}\right)}{2 q_{2}-q_{1}^{2}} \\
k_{0}^{(2)}=\frac{q_{2}^{3}\left(2+q_{2}\right)}{\left(2 q_{2}-q_{1}^{2}\right)^{2}} \quad, \quad k_{1}^{(2)}=\frac{q_{2}^{2}\left(4+2 q_{2}+q_{1}^{2}+q_{2}^{2}\right)}{\left(2 q_{2}-q_{1}^{2}\right)^{2}}
\end{gathered}
$$

and the corresponding forces $M^{(r)}(q)$ are

$$
\begin{gathered}
M^{(-2)}=\frac{1}{q_{2}^{4}}\left(q_{1}\left(3+q_{2}\right), q_{2}\left(4+q_{2}\right)\right)^{T} \\
M^{(-1)}=\frac{1}{q_{1}^{3}}\left(q_{1}, q_{2}\right)^{T} \\
M^{(1)}=\frac{-1}{\left(q_{1}^{2}-2 q_{2}\right)^{2}}\left(q_{1}\left(1+q_{2}\right), q_{2}^{2}\right) \\
M^{(2)}=\frac{q_{2}}{\left(q_{1}^{2}-2 q_{2}\right)^{3}}\left(q_{1}\left(q_{1}^{2}+2 q_{2}^{2}+4 q_{2}+4\right), q_{2}\left(q_{1}^{2}+2 q_{2}^{2}+2 q_{2}\right)\right)^{T}
\end{gathered}
$$


Also in this case the solutions of (40) can easily be calculated:

$$
\lambda_{1}(q)=\frac{\left(q_{2}+2+\sqrt{\Delta}\right) q_{2}}{2\left(q_{1}^{2}-2 q_{2}\right)} \quad, \quad \lambda_{2}(q)=\frac{\left(q_{2}+2-\sqrt{\Delta}\right) q_{2}}{2\left(q_{1}^{2}-2 q_{2}\right)}
$$

with $\Delta=4 q_{1}^{2}+\left(q_{2}-2\right)^{2} \geq 0$. The coordinate curves given by these equations consist of the non-confocal ellipses and hyperbolas [26] and an arbitrary point $\left(q_{1}, q_{2}\right)$ in the $q$-plane may lay not only on intersection of an ellipse and a hyperbola (as it was the case in the classical separability theory) but also on intersection of two ellipses or two hyperbolas. The formulae (42) for $\mu(q, p)$ are in this case too complicated to be presented. The inverse relationships are however still quite compact:

$$
\begin{gathered}
q_{1}=-2 \frac{\sqrt{-\lambda_{1} \lambda_{2}\left(\lambda_{1}+1\right)\left(\lambda_{2}+2\right)}}{\lambda_{1}+\lambda_{2}+\lambda_{1} \lambda_{2}}, q_{2}=-2 \frac{\lambda_{1} \lambda_{2}}{\lambda_{1}+\lambda_{2}+\lambda_{1} \lambda_{2}} \\
p_{1}=2 \frac{\lambda_{1} \lambda_{2}\left(\mu_{1} \lambda_{1}-\mu_{2} \lambda_{2}+\mu_{1}-\mu_{2}\right)}{\lambda_{1}-\lambda_{2}} \\
p_{2}=\frac{-\left(\lambda_{1}+1\right)\left(\lambda_{2}+2\right)\left(\mu_{1}\left(\lambda_{1}^{2} \lambda_{2}-\lambda_{1}^{2}+\lambda_{1} \lambda_{2}\right)-\mu_{2}\left(\lambda_{1} \lambda_{2}^{2}-\lambda_{2}^{2}+\lambda_{1} \lambda_{2}\right)\right)}{\left(\lambda_{1}-\lambda_{2}\right) \sqrt{-\lambda_{1} \lambda_{2}\left(\lambda_{1}+1\right)\left(\lambda_{2}+2\right)}}
\end{gathered}
$$

where we have chosen not to simplify the last expression since

$$
-\lambda_{1} \lambda_{2}\left(\lambda_{1}+1\right)\left(\lambda_{2}+2\right)=q_{1}^{2} q_{2}^{2} /\left(q_{1}^{2}-2 q_{2}\right)^{2}
$$

is always non-negative. Applying these formulas we can express - after long algebraic manipulations - the geodesic Hamiltonians $\gamma_{i}$ in the DN coordinates:

$$
\begin{aligned}
& \gamma_{0}=\frac{-\lambda_{2}}{\lambda_{1}-\lambda_{2}} 4 \lambda_{1}^{2}\left(\lambda_{1}+1\right) \mu_{1}^{2}+\frac{-\lambda_{1}}{\lambda_{2}-\lambda_{1}} 4 \lambda_{2}^{2}\left(\lambda_{2}+1\right) \mu_{2}^{2} \\
& \gamma_{1}=\frac{1}{\lambda_{1}-\lambda_{2}} 4 \lambda_{1}^{2}\left(\lambda_{1}+1\right) \mu_{1}^{2}+\frac{1}{\lambda_{2}-\lambda_{1}} 4 \lambda_{2}^{2}\left(\lambda_{2}+1\right) \mu_{2}^{2}
\end{aligned}
$$

so that $f_{i}\left(\lambda_{i}\right)=4 \lambda_{i}^{2}\left(\lambda_{i}+1\right)$ in this case. The quasi-potentials (53) in the DN coordinates must attain the same form as the quasi-potentials (50)-(51) 
do, namely the form given by (52) since the change of variables (40)-(42) is designed so that the recursion (32) in the DN coordinates always attains the form (45)).

In the end, let us consider a three-dimensional example with matrices $G$ and $\tilde{G}$ chosen as

$$
\begin{gathered}
G=q q^{T}+\left[\begin{array}{lll}
0 & 0 & 1 \\
0 & 1 & 0 \\
1 & 0 & 0
\end{array}\right]=\left[\begin{array}{lll}
q_{1}^{2} & q_{1} q_{2} & q_{1} q_{3}+1 \\
q_{1} q_{2} & q_{2}^{2}+1 & q_{2} q_{2} \\
q_{1} q_{3}+1 & q_{2} q_{3} & q_{3}^{2}
\end{array}\right] \\
\tilde{G}=\left[\begin{array}{l}
0 \\
0 \\
1
\end{array}\right] q^{T}+q\left[\begin{array}{lll}
0 & 0 & 1
\end{array}\right]+\left[\begin{array}{lll}
0 & 0 & 0 \\
0 & 1 & 0 \\
0 & 0 & 0
\end{array}\right]=\left[\begin{array}{lll}
0 & 0 & q_{1} \\
0 & 1 & q_{2} \\
q_{1} & q_{2} & 2 q_{3}
\end{array}\right]
\end{gathered}
$$

The recursion formulas (32) and (33) applied to the geodesic flow $\ddot{q}=0$ yield an infinite sequence of the quasipotentials $k_{\xi}^{(r)}=\sum_{i=0}^{2} k_{i}^{(r)} \xi^{i}, \quad r=$ $\ldots,-1,0,1, \ldots$.Some of them are

$$
\begin{gathered}
k_{0}^{(-1)}=\frac{2 q_{1} q_{3}+2 q_{1}+1}{2 q_{1} q_{3}+q_{2}^{2}+1}, k_{1}^{(-1)}=\frac{q_{1}\left(q_{1}+1\right)}{2 q_{1} q_{3}+q_{2}^{2}+1}, k_{2}^{(-1)}=\frac{q_{1}^{2}}{2 q_{1} q_{3}+q_{2}^{2}+1} \\
k_{0}^{(1)}=\frac{2 q_{1} q_{3}+q_{2}^{2}+1}{q_{1}^{2}}, k_{1}^{(1)}=\frac{2 q_{1} q_{3}+2 q_{1}+1}{q_{1}^{2}}, k_{2}^{(1)}=\frac{2+q_{1}}{q_{1}} \\
k_{0}^{(2)}=\frac{\left(2+q_{1}\right)\left(2 q_{1} q_{3}+q_{2}^{2}+1\right)}{q_{1}^{3}}, k_{1}^{(2)}=\frac{2+q_{1}\left(4 q_{3}+4+2 q_{1}-q_{2}^{2}\right)}{q_{1}^{3}} \\
k_{2}^{(2)}=\frac{q_{1}^{2}-2 q_{1} q_{3}+2 q_{1}+3}{q_{1}^{2}}
\end{gathered}
$$

The corresponding forces $M^{(r)}$ are

$$
\begin{gathered}
M^{(-1)}=\frac{-1}{\left(2 q_{1} q_{3}+q_{2}^{2}+1\right)^{2}}\left(q_{1}^{2}, q_{2}\left(q_{1}+1\right), q_{1} q_{3}-1\right) \\
M^{(1)}=\frac{-1}{q_{1}^{3}}(0,0,1)^{T}
\end{gathered}
$$




$$
\begin{gathered}
M^{(2)}=\frac{-1}{q_{1}^{4}}\left(q_{1}^{2}, q_{1} q_{2}, q_{1} q_{3}+q_{1}+3\right)^{T} \\
M^{(3)}=\frac{-1}{q_{1}^{5}}\left(q_{1}^{2}\left(q_{1}+4\right), q_{1} q_{2}\left(q_{1}+3\right), q_{1}^{2} q_{3}+q_{1}^{2}+3 q_{1}+6\right)^{T}
\end{gathered}
$$

and they fast become complicated with the increasing $|r|$. In this case the formulas (40)-(42) and their inverses are very complicated and can be handled only with the help of a computer algebra package. We will therefore quote here only the formulas for $q(\lambda)$

$$
\begin{gathered}
q_{1}=-2 \frac{1}{\lambda_{1}+\lambda_{2}+\lambda_{3}+1} \\
q_{2}=2 \frac{\sqrt{-\left(\lambda_{1} \lambda_{2} \lambda_{3}+\lambda_{1} \lambda_{2}+\lambda_{1} \lambda_{3}+\lambda_{2} \lambda_{3}+\lambda_{1}+\lambda_{2}+\lambda_{3}+1\right)}}{\lambda_{1}+\lambda_{2}+\lambda_{3}+1} \\
q_{3}=\frac{1}{4} \frac{\lambda_{1}^{2}+\lambda_{2}^{2}+\lambda_{3}^{2}-2\left(\lambda_{1} \lambda_{2}+\lambda_{1} \lambda_{3}+\lambda_{2} \lambda_{3}+\lambda_{1}+\lambda_{2}+\lambda_{3}\right)-3}{\lambda_{1}+\lambda_{2}+\lambda_{3}+1}
\end{gathered}
$$

so that they are built of symmetric polynomials of order three in $\lambda$. The quasi-potentials $k_{i}^{(r)}$ presented above attain in the above DN coordinates the form

$$
\begin{gathered}
k_{0}^{(-1)}=\frac{-\left(\lambda_{1} \lambda_{2}+\lambda_{1} \lambda_{3}+\lambda_{2} \lambda_{3}\right)}{\lambda_{1} \lambda_{2} \lambda_{3}}, k_{1}^{(-1)}=\frac{\lambda_{1}+\lambda_{2}+\lambda_{3}}{\lambda_{1} \lambda_{2} \lambda_{3}}, k_{2}^{(-1)}=\frac{-1}{\lambda_{1} \lambda_{2} \lambda_{3}} \\
k_{0}^{(1)}=-\lambda_{1} \lambda_{2} \lambda_{3}, k_{1}^{(1)}=\lambda_{1} \lambda_{2}+\lambda_{1} \lambda_{3}+\lambda_{2} \lambda_{3}, k_{2}^{(1)}=-\left(\lambda_{1}+\lambda_{2}+\lambda_{3}\right) \\
k_{0}^{(2)}=\left(\lambda_{1} \lambda_{2}+\lambda_{1} \lambda_{3}+\lambda_{2} \lambda_{3}\right) \lambda_{1} \lambda_{2} \lambda_{3} \\
k_{1}^{(2)}=-\left(2 \lambda_{1} \lambda_{2} \lambda_{3}+\lambda_{1} \lambda_{2}^{2}+\lambda_{1} \lambda_{3}^{2}+\lambda_{2} \lambda_{1}^{2}+\lambda_{2} \lambda_{3}^{2}+\lambda_{3} \lambda_{1}^{2}+\lambda_{3} \lambda_{2}^{2}\right) \\
k_{2}^{(2)}=\lambda_{1}^{2}+\lambda_{2}^{2}+\lambda_{3}^{2}+\lambda_{1} \lambda_{2}+\lambda_{1} \lambda_{3}+\lambda_{2} \lambda_{3}
\end{gathered}
$$


which is in accordance with the recursion formula (45). The geodesic Hamiltonians $\gamma_{i}$ have the following structure

$$
\begin{gathered}
\gamma_{0}=\frac{\lambda_{2} \lambda_{3}}{\Delta_{1}} 4\left(1+\lambda_{1}\right) \mu_{1}^{2}+\frac{\lambda_{1} \lambda_{3}}{\Delta_{2}} 4\left(1+\lambda_{2}\right) \mu_{2}^{2}+\frac{\lambda_{1} \lambda_{2}}{\Delta_{3}} 4\left(1+\lambda_{3}\right) \mu_{3}^{2} \\
\gamma_{1}=\frac{-\left(\lambda_{2}+\lambda_{3}\right)}{\Delta_{1}} 4\left(1+\lambda_{1}\right) \mu_{1}^{2}+\frac{-\left(\lambda_{1}+\lambda_{3}\right)}{\Delta_{2}} 4\left(1+\lambda_{2}\right) \mu_{2}^{2}+\frac{-\left(\lambda_{1}+\lambda_{2}\right)}{\Delta_{3}} 4\left(1+\lambda_{3}\right) \mu_{3}^{2} \\
\gamma_{2}=\frac{1}{\Delta_{1}} 4\left(1+\lambda_{1}\right) \mu_{1}^{2}+\frac{1}{\Delta_{2}} 4\left(1+\lambda_{2}\right) \mu_{2}^{2}+\frac{1}{\Delta_{3}} 4\left(1+\lambda_{3}\right) \mu_{3}^{2}
\end{gathered}
$$

so that they have exactly the form (44) with (12) and with $f_{i}\left(\lambda_{i}\right)=4\left(1+\lambda_{i}\right)$.

One can see that in all the above examples the functions $f_{i}\left(\lambda_{i}\right)$ do not depend on $i$, i.e. $f_{i}\left(\lambda_{i}\right)=f\left(\lambda_{i}\right)$.

\section{Conclusions}

In the present article we performed separation of variables for the recently discovered class of quasi-potential systems called bi-cofactor systems. These systems generalize the classical potential systems with additional, quadratic in momenta, integral of motion in the sense that they reduce to these systems in case when one of the matrices $G$ and $\tilde{G}$ is the identity matrix. In this special case the separation formulae (40)-(42) reduce to the well known form the classical separability theory formulas for separation of natural Hamiltonian systems by a point transformation. In the general case, however, these formulas do not have the form of a point transformation and are to our knowledge new in literature. We concluded the article with some non-trivial examples in which the functions $f_{i}\left(\lambda_{i}\right)$ in (12) actually do not depend on $i$ and we can make a conjecture that it is always the case in bi-cofactor systems.

Acknowledgement 19 We would like to thank S. Rauch-Wojciechowski, H. Lundmark and C. Waksjö for interesting discussions during our work on this article. We also thank referees for useful remarks and references. 


\section{References}

[1] Błaszak M, "On separability of bi-Hamiltonian chain with degenerated Poisson structures", J. Math. Phys. 39, 3213 (1998).

[2] Błaszak M, "Bi-Hamiltonian separable chains on Riemannian manifolds", Phys. Lett. A 243, 25 (1998).

[3] Błaszak M, Multi-Hamiltonian Theory of Dynamical Systems, in: Texts and Monographs in Physics, Springer-Verlage (1998).

[4] Błaszak M, "Theory of separability of multi-Hamiltonian chains", $J$. Math. Phys. 40 (1999) 5725.

[5] Błaszak M, "Inverse bi-Hamiltonian separable chains", J. Theor. Math. Phys. 122 (2000) 140.

[6] Błaszak M, "Separability of two-Casimir bi- and tri-Hamiltonian chains", Rep. Math. Phys. 46 (2000) 35.

[7] Błaszak M, "Degenerate Poisson Pencils on Curves: New Separability Theory", J. Nonl. Math.Phys. 7 (2000). 213

[8] Falqui G, Magri F. and Tondo G, "Reduction of bihamiltonian systems and separation of variables: an example from the Boussinesq hierarchy", Theor. Math. Phys. 122 (2000) 176.

[9] Falqui G, Magri F, Pedroni M, "Bihamiltonian geometry and separation of variables for Toda lattices", eprint nlin.SI/0002008 (2000).

[10] Błaszak M, "From bi-Hamiltonian geometry to separation of variables: stationary Harry-Dym and the KdV dressing chain", to appear in Journal of Nonlinear Mathematical Physics (2001).

[11] Marciniak K, Rauch-Wojciechowski S, "Two families of nonstandard Poisson structures for Newton equations", J. Math. Phys. 39 (10), 1998, $5292-5306$.

[12] Rauch-Wojciechowski S, Marciniak K, Lundmark H, "Quasi-Lagrangian systems of Newton equations", J. Math. Phys. 40 (12), 1999, 6366-6398. 
[13] Lundmark H, "Integrable Nonconservative Newton Systems with Quadratic Integrals of Motion", Linköping Studies in Science and Technology. Theses No. 756. Linköping University, 1999.

[14] Lundmark H, "A new class of integrable Newton systems", Journal of Nonlinear Mathematical Physics, 8, (2001) 195-199, Supplement - Proceedings of NEEDS '99, Kolymbari, Crete.

[15] Marciniak K, Rauch-Wojciechowski S, "Integrable perturbations of the harmonic oscillator and Poisson pencils", Inverse Problems 17, 2001, 191-209.

[16] Eisenhart L. P., "Separable systems of Stäckel", Ann. Math. 35 (1934) pp. 284-305.

[17] Benenti S, Intrinsic characterization of the variable separation in the Hamilton-Jacobi equation, J. Math. Phys. 38 (12), 1997, pp. 6578-6602.

[18] Ibort A, Magri F., Marmo G, "Bihamiltonian structures and Stäckel separability", Journal of Geometry and Physics 33 (2000) pp. 210-228.

[19] Morosi C, Tondo J, "Quasi-bihamiltonian systems and separability", J. Math. Phys. 38 , 1997, pp. 6578-6602.

[20] Kalnins E G, Miller W Jr., Killing tensors and variable separation for Hamilton-Jacobi and Helmholtz equations", SIAM J. Math. Anal. 11 (6), 1980, pp. 1011-1026.

[21] Crampin M, Sarlet W, " A class of non-conservative Lagrangian systems on Riemannian manifolds", J. Math. Phys. 42 (9), 2001, pp. 4313-4326.

[22] Magri F, "A simple model of the integrable Hamiltonian equation", J. Math. Phys. 19 (5), 1978, pp. 1156-1162.

[23] Lundmark H, "Newton systems of cofactor type in Euclidean and Riemannian spaces", Linköping Studies in Science and Technology. Dissertations No. 719. Linköping University, 2001.

[24] Falqui G., Magri F., Pedroni M., Zubelli J.P., "A Bi-Hamiltonian Theory for Stationary KdV flows and their Separability", RegularandChaoticDynamic 5 (2000), pp. 33-52. 
[25] Ramani A, Dorizzi B, Grammaticos B, "Painleve conjecture revisited", Phys.Rev.Lett. 49 (1982), pp. 1539-1541.

[26] Lundmark H, private communication. 